\title{
Testicular Cyst
}

National Cancer Institute

\section{Source}

National Cancer Institute. Testicular Cyst. NCI Thesaurus. Code C3885.

A cystic lesion located in the testes. 